\title{
La escuela y los alumnos con grave retraso en el desarrollo
}

\author{
Javier Tamarit
}

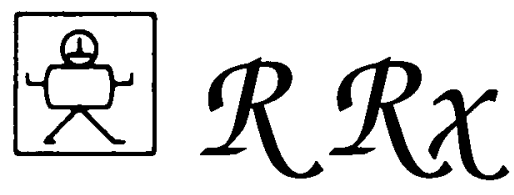

Ante el desafío que plantea la escolarización de los niños con grave retraso mental, los autores de este trabajo proponen los tres objetivos educativos sobre los que configurar la enseñanza, así como los medios sociales e instrumentales que deben apoyar la estrategia educativa.

\section{INTRODUCCION}

La educación de niños y niñas con graves retrasos en su desarrollo supone un desafio profesional y social importante. Frente a las actitudes que se enfrentan al mismo con desánimo y con pesimismo, considerando que la tarea educativa en estas personas es prácticamente inútil, y que lo único posible y deseable es una actuación asistencial en la que se asegure el máximo bienestar, queremos manifestar aquí nuestra defensa infranqueable de una actitud optimista que considera que la educación de estas personas es posible y es una tarea ineludible. En este trabajo expondremos nuestro planteamiento educativo, pero previamente vamos a dedicar un breve espacio a la descripción de la población a la que nos referimos cuando hablamos de alumnos y alumnas con grave retraso en su desarrollo.

¿Quiénes configuran el alumnado con grave retraso en el desarrollo?:

Este grupo no configura una categoría de límites concretos y claros, en los que se perciba sịn duda la inclusión o exclusión de sus miembros, sino que determina, más bien, una categoría de límites borrosos, en la que no está tan claro qué personas pertenecen a este grupo y por qué razones y qué personas no pertenecen al mismo. En general, aquí vamos a considerar que el alumnado con grave retraso en el desarrollo se caracteriza por tener un rango de retraso mental grave o profundo $(C I=20 / 25$ a $35 / 40$, en el caso de grave; y $\mathrm{CI}=$ por debajo de $20 / 25$, en el de profundo, según la DSMIII-r, 1987), junto con un perfil, en una o varias de las áreas básicas del desarrollo (social, comunicación, conducta), que muestra alteraciones que no se explican mediante el nivel de desarrollo. Además, se pueden dar otros trastornos asociados, tales como déficits sensoriales (ceguera, sordera) o alteraciones motrices. En resúmen, personas que tienen retraso mental importante y algo más que no se explica por dicho retraso. Dentro de este grupo amplio entrarian las personas con retraso mental y autismo, u otros trastornos generalizados del desarrollo, en las que se da 
una desviación importante con respecto al modelo normal de desarrollo en sus patrones de interacción social recíproca, de comunicación, tanto verbal como no verbal, y en las que se manifiesta un patrón de intereses y actividades notoriamente restringido y estereotipado.

\section{VALORACION DE LAS NECESIDADES EDUCATIVAS}

Las necesidades educativas especiales que este tipo de alumnado presenta revisten el carácter de graves y permanentes. No obstante, debería tenerse siempre en cuenta la tremenda variabilidad y heterogeneidad que existe dentro del grupo, lo que conlleva perfiles psicológicos diferenciados que requieren acercamientos valorativos individualizados. Con estos alumnos y alumnas es especialmente importante valorar no solo sus necesidades educativas especiales, sino tambien valorar sus necesidades sanitarias y sociales, que tambien son especiales, graves y permanentes, puesto que esas necesidades van a influir en la respuesta educativa que se ofrezca. Hemos de pensar que un porcentaje amplio toma medicación de forma permanente, que otro porcentaje importante presenta trastornos de tipo epiléptico, y que prácticamente todos tienen revisiones médicas anuales en las que la información desde un contexto escolar debería ser una pieza de valoración importante. Por otro lado, estos alumnos y alumnas tienen una serie de condiciones o características que hay que tener en cuenta para entender mejor la actuación educativa. Son personas que van a necesitar apoyo permanente; además, y sobre todo cuanto más edad tienen, son personas que generan a primera vista un menor enganche social-emocional-afectivo en los demás; en tercer lugar, son menos manejables por medio de la palabra y necesitan, por tanto, un mayor manejo físico. Todo ello conlleva un mayor cansancio físico en las personas que se preocupan de su educación, quienes además perciben que su tarea tiene una gratificación personal importante pero lenta y costosa. Por tanto, en la valoración de las necesidades de este tipo de alumnado, hay que incidir también en la valoración de las necesidades de los profesionales de la educación con los que están, propiciando suficiente personal, y sistemas de organización y de formación de ese personal idóneos para la creación y el funcionamiento sostenido de equipos eficaces de intervención educativa.

\section{CRITERIOS PARA LA ESCOLARIZACION}

Por regla general el alumnado con grave retraso en el desarrollo serán escolarizados en Centros específicos con un número reducido de alumnos por aula, y con un número elevado de profesionales con especialización (logopedas, fisioterapeutas, trabajadores sociales, psicólogos o pedagogos). No obstante, no conviene olvidar que la escolarización en Centros específicos no supone la exclusión de entornos normalizados, sino que, por el contrario, exige una mayor atención a la planificación de actividades de movilidad comunitaria aprovechando los recursos propios de la comunidad y favoreciendo la relación con los demás en esos entornos normalizados. En este sentido no está de más hacer hincapié en que la integración plena es un proceso de enseñanza-aprendizaje (la integración se aprende y se enseña, no surge de la nada) y es un proceso recíproco (tanto se ha de integrar el niño o la niña con grave retraso en su desarrollo con las personas normales, como éstas con aquellos).

\section{OBJETIVOS Y CONTENIDOS PRIORITARIOS DURANTE LA ESCOLARIZACION OBLIGATORIA}

La respuesta educativa ha de apoyarse imprescindiblemente en un marco de realidad, $y$, en este sentido, ha de ajustarse a: a) la realidad del alumnado (teniendo en 
cuenta sus edades cronológicas y.de desarrollo, sus intereses, sus perfiles de competencia física, sensorial, mental, etc.); b) la realidad de la comunidad educativa (buscando que la tarea educativa sea percibida y considerada con un alto grado de validez social); y c) la realidad de la política educativa (adoptando y adaptando el espíritu y la filosofía que rigen esa política a las necesidades del alumnado con graves retrasos en su desarrollo).

En la planificación educativa de estos alumnos, las áreas social (cognitivosocial y socio-emocional) y comunicativa (comunicarivo-social) son el núcleo central a donde se dirige la intervención. Las demás áreas son también, no cabe duda, importantes, pero en cuanto a las repercusiones que tengan en estas. En este sentido, merece la pena recalcar que la construcción del desarrollo en el niño proviene del control y manipulación que ese niño sea capaz de ejercer sobre el entorno social, y con el entorno social, y no solo del que pueda ejercer sobre el entorno físico, y con el entorno físico. Además de estas áreas básicas, planteamos que otro objetivo básico de la actuación educativa es propiciar los máximos niveles de independencia personal y social de cara a lograr el mayor desenvolvimiento en los entornos menos restrictivos posibles. Para acercarse a este objetivo es especialmente relevante el considerar prioritaria la enseñanza de competencias básicas de autocontrol del propio comportamiento, de manera que las acciones del alumno o alumna, ajustadas al contexto, se lleven a cabo a través de mediadores manejados y controlados por ellos. Esto implica una consideración del autocontrol como una competencia que se construye progresivamente en el desarrollo, por tanto una competencia con diferentes niveles de adquisición, y no como el resultado de unas capacidades cognitivas previas. Así, es posible hablar de autocontrol y hablar de la enseñanza del autocontrol en poblaciones que, como las que aquí comentamos, tienen grave retraso mental.

Las vigas maestras sobre las que se sustenta la elección de objetivos educativos, y su puesta en marcha, son el conocimiento preciso de los mecanismos esenciales en la construcción del desarrollo normal, la funcionalidad de los contenidos, el que esos contenidos sean en sí motivantes, y el que se vayan logrando, por medio del propio proceso educativo, ejecuciones espontáneas de los aprendizajes adquiridos. Es decir, es preciso enseñar la habilidad, funcional y motivante, pero es preciso enseñar también el uso, ajustado al contexto, de la habilidad.

Vamos a exponer a continuación tres puntos que pensamos que son especialmente importantes y prioritarios en esta población: la eliminación de barteras psicológicas; la actuación ante graves trastornos de conducta; y la consideración de la familia como elemento importante en la programación educativa.

1. Eliminación de barreras psicológicas: En el desarrollo normal, los niños son competentes para, de una manera natural, extraer del entorno físico los estímulos relevantes, aquellos con significado funcional, obviando los estímulos inútiles o interfirientes. Asímismo, son competentes para entender las claves que regulan el entorno social, el comportamiento interpersonal. Estas claves se caracterizan por ser sutiles - de tenue percepción-, complejas — compuestas por varios estimulos a la vez-, pasajeras —de rápido desvanecimiento en el tiempo-y variadas —diferentes, aún ligeramente, de una ocasión a otra- Los alumnos y alumnas con graves retrasos en el desarrollo tienen problemas para entender claves con esas características. Las dificultades que presentan en abstraer información de las claves del entorno físico normal, tanto en cuanto a claves de orientación espacial, como, especialmente, en cuanto a claves de orientación temporal, hacen que sea prioritaria la tarea de adaptar las claves existentes, o crear unas nuevas adecuadas a sus niveles de competencia. Esto implica que se debe estructurar el Centro (tanto a nivel físico como a nivel social - p.e., mediante la coherencia en la actuación de los profesionales-) de manera que estos alumnos y alumnas puedan manejarse adecuadamente y cuenten 
con el necesario control del medio que les permita avanzar en su desarrollo y en su independencia. Se trata, por tanto, de eliminar todas aquellas barreras cognitivas que, por la dificultad de las claves que poseen, impiden manejar eficazmente el entorno, al igual que ocurre en el caso de las barreras arquitectónicas. Es especialmente necesario que se pongan todos los medios al alcance para que se transmitan al hogar estos mismos planteamientos.

2. Actuando ante problemas graves de conducta: Las graves problemáticas conductuales que los alumnos o alumnas pueden presentar (agresión, autolesión, etc.) han de ser minuciosamente analizadas, mediante un análisis funcional contextualizado para su mejor comprensión, y para la determinación del tratamiento adecuado. El tratamiento deberá ser mediante sistemas de intervención positivos, que tiendan a favorecer capacidades de autocontrol conductual. En casos extremos estos planteamientos pueden no ser completamente satisfactorios. En estos casos se requiere una colaboración estrecha entre los servicios médicos y los educativos. Si las conductas revisten grave riesgo para la propia persona o para los demás y no han podido ser controladas mediante tratamientos positivos se deberán analizar las posibilidades de sistemas de restricción física, como medida temporal. En casos muy extremos se puede considerar ineludible la aplicación de sistemas de carácter aversivo. En este caso, se tendrá especialmente en cuenta el respeto a la dignidad de la persona por encima de todo, se requerirá un consenso profesional, el permiso explícito de la familia, y que el tratamiento sea llevado a cabo en presencia de observadores que evaluen la adecuación de la intervención al programa propuesto. Las familias han de estar siempre informadas con absoluto detalle de las propuestas, razonadas, de intervención.

3. Consensuando objetivos: Es necesario favorecer la existencia de un consenso entre las familias y los profesionales en cuanto a que los objetivos educativos planteados sean considerados válidos e importantes. La existencia de esta validez social en cuanto a la elección de objetivos conllevará una relación familia-Centro más armónica, realista y motivadora. Esto, sin duda, repercutirá en la generalización del trabajo educativo a la casa, y, por tanto, en la mejora significativa de los alumnos. Esta búsqueda de objetivos consensuados es difícil, por lo que se hace necesario diseñar programas que persigan el conocimiento detallado de las necesidades familiares en los distintos segmentos de edad (niñez, adolescencia,...).

Vamos a considerar dentro de este apartado la orientación familiar. Esta orientación ha de contemplarse como un servicio amplio y flexible destinado a afrontar y resolver las necesidades variadas que las familias puedan tener, tales como: a)necesidad de orientación y apoyo psicológico; b) necesidad de información sobre la actuación educativa a seguir con su hijo o hija; y c) necesidad de contar con servicios de respiro y apoyo familiar, para frenar el proceso de insularización que se origina a raiz de la reducción de relaciones sociales externas a la familia, a causa de la dedicación casi exclusiva al hijo o hija.

\section{ESTRATEGIAS METODOLOGICAS Y ORGANIZATIVAS MAS FAVORABLES}

El aula, como entorno significativo de ocurrencia del aprendizaje, solo tiene cierto sentido cuando los contenidos de aprendizaje son abstractos y cuando los alumnos y alumnas tienen una competencia cognitiva tal que les permite procesar ese conjunto de contenidos a través de procesos mentales superiores.

En los alumnos y alumnas en los que sus competencias cognitivas no les permiten manejar complejas representaciones mentales, en los que sus capacidades no les permiten entender contenidos de alto valor símbolico, y, además, en los que nos 
podemos plantear incluso si esos conocimientos —abstractos y simbólicos- serían los más adecuados para mejorar su desarrollo, la organización a través de aulas típicamente escolares puede cuestionarse.

Por otro lado, y como ya señalábamos anteriormente, el objeto de la educación lo constituye el conjunto de estrategias que permiten un control, una comprensión y una regulación adecuadas del entorno físico, del entorno social y del entorno «interno" (esto es, el control, comprensión y regulación de sí mismo). En el tipo de alumnado al que nos estamos refiriendo este objeto se traduce, principalmente, en la necesidad de enseñar estrategias de interacción social, en enseñar comprensión de las claves socioemocionales, enseñar sistemas de autorregulación eficaz de la propia conducta y enseñar estrategias de comunicación interpersonal (bien sea a través de signos, de habla, de pictogramas, de acciones básicas, etc.).

Entendemos que en estos alumnos y alumnas, que presentan graves alteraciones del desarrollo e importante retraso mental, el aprendizaje de las estrategias antes mencionadas se genera a través de la acción - guiada - en entornos naturales y motivadores, y a través del diseño cuidadoso de consecuencias funcionales de esa acción. Entendemos, también, que las tareas para la enseñanza de habilidades sociales, comunicativas y de autorregulación no pueden ceñirse a tiempos concretos (no es real decir que se enseña comunicación de $10.00 \mathrm{~h}$. a $10.30 \mathrm{~h}$.), sino que sabemos, por nuestra propia experiencia, que el proceso de enseñanza es eso, un proceso, y que es permanente. Un proceso permanente en el que es de la máxima importancia la consideración de que ese proceso es una construcción continuada del desarrollo, que realiza el alumno o la alumna bajo la guía del adulto, y que para que esa construcción se realice es necesario que ese adulto se dé cuenta de que él es una pieza clave en el proceso y no un mero espectador externo transmisor, más o menos eficaz, de conocimientos.

La programación por Entornos disminuye los problemas de generalización, en el sentido de que en un aula prototípica hay un recorrido grande entre ese aula, como lugar donde se obriene el aprendizaje, y el lugar natural donde ese aprendizaje es funcional, adecuado y necesario.

Los Entornos Educativamente Significativos - que son las verdaderas aulas, en cuanto que son los espacios físicos en los que se desarrolla el proceso de enseñanzaaprendizaje - son todos los espacios de la Escuela y los espacios relevantes del exterior de la misma (p.e., aulas, comedor, aseos, patio, gimnasio, campo de deporte, tienda de ultramarinos, piscina, etc.). En cada uno de esos Entornos no se enseña solo lo que supuestamente es específico de ese Entorno (por ejemplo, en el aseo habilidades de autonomía tales como lavarse las manos, o control de esfínteres), sino que además de esos objetivos específicos se enseñan de manera prioritaria los objetivos comunicativos, sociales, conductuales, cognitivos, etc. que ban sido previamente programados. Es decir, cada Entorno de Aprendizaje es lugar de un rico y variado potencial educativo.

La unión de Entornos Educativamente Significativos con criterios de agrupamientos flexibles, en un clima de funcionalidad, motivación positiva y en un clima favorecedor de la espontaneidad, configura la estructura básica, organizativa y técnica en la que ofrecer la enseñanza de las diversas estrategias sociales, comunicativas, de regulación conductual, de independencia y autonomía, motrices, cognitivas, etc., que, a través de contenidos y actividades concretas, se dirigen a la activación de los procesos básicos relevantes que propician el proceso global de construcción del desarrollo.

\section{CRITERIOS Y PROCEDIMIENTOS PARA LA EVALUACION}

Se plantea una evaluación contínua e individualizada que sea efectiva en cuanto al desarrollo de nuevas programaciones y en cuanto a la crítica de las que se hayan llevado a cabo. Podemos considerar cuatro niveles de evaluación: 
1. Evaluación global psicológicalpedagógica: Esta evaluación puede ser realizada por el Servicio de Orientación del Centro. Es individualizada y su objetivo es analizar el desarrollo de competencias generales. Este análisis, que debería efectuarse en equipo, tendría que contar con los datos de los restantes niveles de evaluación.

2. Evaluación específica educativa: Esta evaluación puede ser realizada por la persona responsable del aula junto con aquellas otras personas profesionales que estén en contacto directo con el alumno o alumna. Su objetivo será el analizar el desarrollo de las habilidades específicas que se trabajan de acuerdo a la programación individual. Esta evaluación es de carácter cualitativo y cuantitativo, y no se haría basándose en criterios de tiempo sino de adquisición. El análisis de los datos obtenidos, como en el caso anterior, debería efectuarse en equipo y contando con los datos de los restantes niveles de evaluación.

3. Evaluación de la valoración social de la actuación educativa: Debería efectuarse en equipo, teniendo en cuenta los datos aportados por los familiares y los datos aportados por los profesionales de cada Centro. Generalmente será una evaluación cualitativa, que junto con los otros niveles perfile y corrija la actuación educativa. La información contrastada de las familias y del Centro permite hacer correcciones en los criterios de programación, lo cual obviamente redunda en planteamientos educativos más efectivos y con mayor grado de validación social.

4. Evaluación global de la marcha del Centro: Se plantea la necesidad de una evaluación continuada de la marcha global de cada Centro. Esta evaluación y valoración debería ser un proceso abierto y dinámico, de discusión y crítica constructiva, cuyo objetivo resida en la corrección y mejora de la organización y planteamiento de la actuación educativa y del sistema de funcionamiento del Centro.

\section{CONCLUSION}

En este trabajo hemos planteado un modo de afrontar la escolarización de alumnos y alumnas con graves retrasos en su desarrollo. Pretende ser solo una opción, necesariamente discutible, y no un modelo cerrado de actuación. El modelo eficaz lo iremos construyendo entre todos, paso a paso, con la experiencia diaria. Profesionalmente, socialmente y personalmente, la tarea merece la pena.

\section{Referencias}

AMERICAN PSYCHIATRIC ASSOCIATION (1987): Diagnostic and Statistical Manual of Mental Disorders. 3ed ed. rev. Washington, DC: Author. (Edición española, Ed. Masson, 1988).

KIERnAN, C., JORDAN, R. y SAUNDERS, C. (1978): Starting off. London: Souvenir Press (Trad. cast.: Cómo conseguir que el niño juegue y se comunique. Madrid: INSERSO. 1983).

MnEs, M. (1988): «La deficiencia mental profunda: el último desafio». Siglo Cero, 116, Marzo-Abril, 16-56.

MiTtLER, P. (1988): «La ayuda a las personas con deficiencia profunda: Panorámica General». Siglo Cero, 116, Marzo-Abril, 12-15.

TAMARIT, J. (1988): «Los trastornos de la comunicación en deficiencia mental y otras alteraciones evolutivas: intervención mediante sistemas de Comunicación Total». En C. Basil y R. Puig (Eds.): Comunicación Aumentativa. Madrid: INSERSO

TAMARIT, J. (1989): «Uso y abuso de los Sistemas Alternativos de Comunicación”. Comunicación, Lenguaje y Educación, 1, 81-94

VARIOS AUTORES (1989): Intervención educativa en autismo infantil. Madrid: Centro Nacional de Recursos para la Educación Especial.

VARIOS AUTORES (1991): Zerbitzuan (Revista de Servicios Sociales), 17 (monográfico), Noviembre. 


\section{Bibliografía básica. Sugerencia para los lectores}

El Módulo sobre Intervención educativa en autismo infantil, editado por el Centro Nacional de Recursos para la Educación Especial, y que consta de diez cuadernos (uno por tema) más un video explicativo, es un material de referencia básico para el trabajo con el alumnado al que nos hemos referido.

La Revista de Servicios Sociales del Consejo Vasco de Bienestar Social, correspondiente a Noviembre de 1991, está dedicada, en su totalidad, a «Los problemas de comportamiento en Personas con Retraso Mental Grave».

El libro de Kiernan, editado por el INSERSO, y titulado «Como conseguir que el niño juegue y se comunique», puede ser un buen auxiliar para la elección adecuada de objetivos.

La lectura del artículo sobre «Deficiencia Profunda: el último desafio», en la Revista de Siglo Cero, número 116 puede ser útil en proveer una visión global del problema.

Mis artículos «Los trastornos de la comunicación en deficiencia mental y otras alteraciones evolutivas: intervención mediante sistemas de comunicación total"” en el libro Comunicación Aumentativa de C. Basil y R. Puig (Eds.), editado por el INSERSO, y «Uso y abuso de los sistemas alternativos de comunicación», en la Revista Comunicación, Lenguaje \& Educación, número 1 (1989) pueden ser un punto de partida para el empleo de Sistemas Alternativos de Comunicación.

La escuela y los alumnos con grave retraso en el desarrollo

Javier Tamarit

$C L \& E, 1994,22, p p .47-53$

Resumen: En este artículo se plantean las bases para la intervención educativa en alumnos con graves retrasos en su desarrollo. Estas bases pueden resumirse así: a) La enseñanza de estrategias para el control y manejo del entorno, tanto del entorno físico como, predominantemente, del entorno social, a través de la enseñanza de habilidades sociales, comunicativas (con lenguaje oral o con el empleo de sistemas alternativos de comunicación) y de autorregulación de la conducta; y b) la adaptación del entorno mediante la eliminación de barreras psicológicas o cognitivas. Se propone un modelo de actuación educativa que esté basado en el aprovechamiento de entornos naturales de aprendizaje y que considere a la familia como un elemento primordial en el diseño del programa educativo.

Datos sobre el autor: Licenciado en psicología. Director del equipo CEPTI desde 1979. Accualmente presidente de AETAPEI (Asociación española de terapeutas de autismo y psicosis infantiles).

Dirección: CEPRI. Avda. de la Victoria, 63, 28023 Madrid.

C PERMISOS PARA CITAR O REPRODUCIR EN OTRAS FUENTES: Se pueden citar libremente hasta 500 palabras. Para reproducir una porción de texto mayor, figuras o ilustraciones, se deberá pedir permiso por escrito a la revista, especificando el uso al que se destina el texto. En todos los casos, se deberá citar el copyright de $C L \& E$. En el caso de artículos o textos que hayan sido a su vez reproducidos en $C L \& E$ los interesados deberán dirigirse tanto a los detentadores del copyright original como a $C L \& E$, en el caso de que se quiera hacer uso de la traducción. FOTOCOPIAS: Para todo lo relacionado con el uso mediante fotocopia del material de esta revista, deberán dirigirse a: CEDRO, C/ José Marañón, 10, 3. Izda. Tel. 5941575 . Fax 4453567 


\section{Dossier documental}

Se ofrecen a continuación una selección de referencias bibliográficas que, tal como se se ha señalado al principio, complementan la información vertida en los diferentes trabajos presentados y que, por lo tanto, han de permitir a los profesionales interesados ampliar sus conocimientos acerca de cuestiones que no han podido ser tratadas con suficiente amplitud por los autores.

Las referencias se presentan agrupadas según las distintas condiciones personales de hándicap presentes en los alumnos, haciendo así más fácil y útil su consulta.

Los criterios que han guiado la selección de estas referencias han sido los siguientes. En primer lugar, se pretende ofrecer una bibliografía básica, donde prima la pertinencia por encima de la amplitud.

En segundo lugar, se opta por centrarse en los retos que el proceso educativo de estos alumnos conlleva para los profesores y otros profesionales, renunciando a aspectos de naturaleza distinta que quizás desde otras prioridades pudieran ser asimismo considerados.

Por último, se ha tenido en cuenta que sean de fácil acceso y manejo por parte de los posibles usuarios.

\section{DÉFICIT AUDITIVO}

No resulta fácil elaborar una bibliografía básica centrada en la educación de los alumnos sordos. La mayor parte de las publicaciones existentes tienen un carácter más especializado y se centran en el ámbito lingüístico/comunicativo y más concretamente en la intervención logopédica. Se proponen al lector un conjunto de referencias que, tomadas globalmente, pueden ayudar a establecer una visión general de cuestiones relativas al desarrollo cognitivo, comunicativo y socio-afectivo de estos alumnos junto a consideraciones educativas.

ALONSO, P., y VALMASEDA, M. (en prensa). Los sistemas de comunicación sin ayuda. En M. Sotillo, Los sistemas de comunicación. Madrid: Ediciones Trotta.

GARRIDO, C.; VÁSQUEZ, A., y MARTíneZ, I. (1990). La integración de los niños sordos en el sistema escolar. El caso de Adela G. Infancia y Aprendizaje, 50, 43-62.

LUCAS, C. (1989). The sociolinguistics of the Deaf Community.San Diego: Academic Press.

MARCHESI, A. (1987). El desarrollo cognitivo y linguístico de los niños sordos. Madrid: Alianza Psicología.

MARTíneZ, I. (1990). El desafío de la Integración. Barcelona: Ediciones Milán y Fundació Catalana per a la Síndrome de Down.

Neal Davies, S. (1991). The transition toward Bilingual education of deaf children in Sweden and Denmark: Perspectives on Language. Sign Language Studies, 71, 169-195.

SANDERS, D. (1988). Teacbing deaf children: Techniques and methods. Boston: College-Hill Publication.

StONe HARRIS, R. (1988). Let's learn about deafness. Washington D.C.: Gallaudet Press. (Traducción española (1992), Aprendiendo cosas sobre la sordera: actividades para el aula. Madrid: Centro Nacional de Recursos para la Educación Especial. M.E.C.

VV.AA. (1991). Las necesidades educativas especiales del niño con deficiencia auditiva. Madrid: M.E.C. -Centro Nacional de Recursos para la Educación Especial. Serie Formación.

\section{DÉFICIT VISUAL}

El conjunto de referencias que se ofrecen tienen un marcado carácter práctico sin excluir un referente conceptual sólido. Fundamentalmente se trata de manuales para la valoración educativa y para la estimulación de la visión de los niños con gra- 


\section{¿Conoces ya Socio-Cultural Research $\mathcal{N}$ ews? \\ Si quieres recibir el primer número para conocerlo, solicítalo sin compromiso a Ca Fundación Infancia y Aprendizaje. Carretera de Canillas, 138, of. 16 C. 28043 Madrid}

\begin{tabular}{|c|c|}
\hline & $\begin{array}{c}\text { STAFF } \\
\text { Editorial Board } \\
\text { Editors } \\
\text { Pilar Lacasa (UNED, España) \& } \\
\text { Jacqueline Baker-Sennett (University of } \\
\text { British Columbia, Canadá) }\end{array}$ \\
\hline & $\begin{array}{c}\text { Associate Editors } \\
\text { Amelia Alvarez, Pablo del Río, } \\
\text { Bernard Schneuwly, Jaan Valsiner, } \\
\text { James V. Wertsch }\end{array}$ \\
\hline & Consulting Editors \\
\hline & $\begin{array}{c}\text { Jean Paul Bronckart } \\
\text { (Université de Genève, Switzerland) }\end{array}$ \\
\hline & $\begin{array}{c}\text { Michel Brossard } \\
\text { (Université de Bordeaux II, France) }\end{array}$ \\
\hline & $\begin{array}{c}\text { Michael Cole } \\
\text { (University of California, USA) }\end{array}$ \\
\hline & $\begin{array}{c}\text { César Coll } \\
\text { (Universidad de Barcelona, España) }\end{array}$ \\
\hline & $\begin{array}{c}\text { Boris Elkonin } \\
\text { (University of Moscow, Russia) }\end{array}$ \\
\hline & $\begin{array}{c}\text { Nada Ignjatovic-Savic } \\
\text { (Beograd University, Yugoslavia) }\end{array}$ \\
\hline & $\begin{array}{c}\text { Karin Junefelt } \\
\text { (University of Stockholm, Sweden) }\end{array}$ \\
\hline & $\begin{array}{c}\text { Luis Moll } \\
\text { (University of Arizona, USA) }\end{array}$ \\
\hline & $\begin{array}{c}\text { Juan Daniel Ramirez } \\
\text { (Universidad de Sevilla, España) }\end{array}$ \\
\hline & $\begin{array}{c}\text { Elsie Rockwell } \\
\text { (Centro de Investigaciones y de Estudios } \\
\text { Avanzados, México) }\end{array}$ \\
\hline & $\begin{array}{c}\text { Cintia Rodriguez } \\
\text { (Universidad Autónoma Madrid, España) }\end{array}$ \\
\hline & $\begin{array}{c}\text { Barbara Rogoff } \\
\text { (University of Utah, USA) }\end{array}$ \\
\hline & $\begin{array}{c}\text { Alberto Rosa } \\
\text { (Universidad Autonoma Madrid, España) }\end{array}$ \\
\hline & $\begin{array}{c}\text { Ana Smolka } \\
\text { (Universidade Stadual de Campinas, Brazil) }\end{array}$ \\
\hline & $\begin{array}{c}\text { Nobumoto Tajima } \\
\text { (Tokyo University of Foreign Snidies, Japan) }\end{array}$ \\
\hline & $\begin{array}{c}\text { Henry Trueba } \\
\text { (University of Winsconsin, USA) }\end{array}$ \\
\hline & $\begin{array}{c}\text { Peeter Tulviste } \\
\text { (University of Tartu, Estonia) }\end{array}$ \\
\hline & $\begin{array}{c}\text { Serena Vegetti } \\
\text { (Università degli Studi di Roma, Italy) }\end{array}$ \\
\hline & $\begin{array}{c}\text { Gordon Wells } \\
\text { (Ontario Inst. Studies in Education, Canada) }\end{array}$ \\
\hline
\end{tabular}

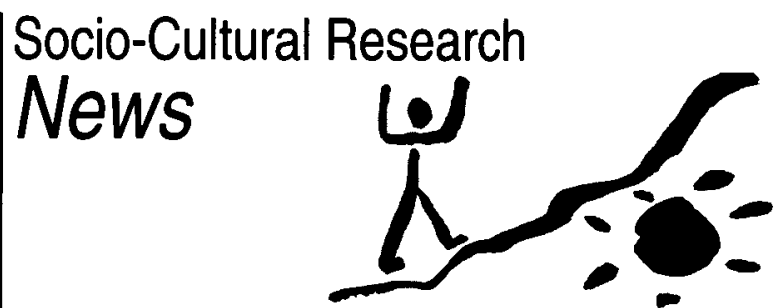

The Socio-Cultural Research News is a biannual publicacion from Infancia $y$ Aprendizaje Foundation for the Socio-Cultural Research International Network.

Each edition of Sociocultural News will highlight theoretical empirical works of one researcher or line of research. If you are interested in submitting your work to Sociocultural News, please send a diskette containing comments, abstracts of your research, theoretical reflections or books reviews (no more than 3 pages single spaced) to one of the following addresses:

El Socio-Cultural Research News es una publicación de la Fundación Infancia y Aprendizaje para la Red Internacional de Investigadores en la perspectiva SocioCultural. Se admiten contribuciones en tres idiomas: español, francés e inglés. E! formato de las contribuciones debe ser breve $(3$ holandesas a un espacio o equivalente) y versar sobre líneas de investigación en curso, comentarios sobre temas teóricos o aplicados, y revisiones de bibliografía. Los autores deben enviar su texto en diskette de 3,5 pulgadas (PC o Macintosh, Word, Word Perfect, Write Now o Mc Write) debidamente etiquetados y especificando el tipo de procesador de texto o de programa gráfico empleado. Deberá adjuntar igualmente tres copias impresas a cualquiera de las directoras cuya dirección se especifica.

Pilar Lacasa,

Facultad de Psicología. U.N.E.D.

Dpto. Psicología Social y Evolutiva. Ciudad Universitaria.

Madrid 28071. Spain

7734-1-3986244; FAX 34-1-3986234 ; E-mail: pilar.lacasa@uned.es

Jacquelyn Baker-Sennett,

University of British Columbia

Faculty of Education- EPSE, Vancouver, B.C. Canada, V6T 124

or U.S. Address 4796 Lincoln Road Blaine, WA 98230

7 604-822-9223; FAX: 604-822-3302; E-mail: userjac@ubc.mtsg.ca

Manuscripts in three copies should be submitted either in french, spanish or english, but the correctness in each of them is of the entire responsibility of the author.

The text should be composed using one of the following word-processing programs: - IBM Comparible PC: "Word Perfect" or "Word"

- Macintosh: "Word", "Word Perfect", "Write Now" or "Mac Wrice"

A 3,5" diskette, properly labeled including your name, the names of the files containing the text and graphics (if applicable), as well as the computer word processor and graphics program used.. Three printed copies of the text should also be provided.

\section{PLEASE, SEND NOW SUBSCRIPTION FORM}

Price: 1.500 pts. (1994-1995, two volumes, four issues) (For air mail, add on 500 pts.)

Name:

Mailing address:

Form of Payment: $\square$ Cheque (only convertible pesetas)

$\square$ MasterCard or $\square$ VISA . Card $\mathbf{n}^{\circ}$ :

Exp. date.

Signature 
ves trastornos visuales y de guías para su educación. Asimismo se incluyen trabajos, en forma de ponencias, presentados a distintas Jornadas; todos ellos recogen experiencias de profesionales en la educación de estos alumnos.

Chapman-Tobin (1986). Mira y Piensa. Madrid: ONCE.

LEONHARDT, M. (1992). El bebéciego. Barcelona: MASSON-ONCE.

Barraga, N. (1986). Textos reunidos de la Dra. Barraga. Programa para desarrollar eficiencia en el funcionamiento visual. Madrid: ONCE.

Guinea, C., TubaU, G., Esteban, M." A. (1983). La escuela abierta al niño ciego. Barcelona: Fundació "La Caixa".

FraiberG, S. (1980). Niños Ciegos. Madrid: INSERSO.

VV.AA. (1986, 1987, 1988). Jornadas sobre educación de los niños ciegos y deficientes visuales. Madrid: ONCE. TOBIN, J. M. (1986). Algunos aspectos del desarrollo cognoscitivo en los niños ciegos. Barcelona: Fundació "La Caixa".

\section{DÉFICIT MOTRIZ}

El lector interesado encontrará a continuación una selección de textos que orientarán su actuación en los principales ámbitos que configuran el proceso educativo de los alumnos con déficit motriz. Por un lado, la valoración de las necesidades educativas junto con las ayudas técnicas más adecuadas; por otro lado, las actividades de enseñanza y aprendizaje, con especial referencia al papel que puede cumplir el juego, junto con el uso adecuado de los sistemas aumentativos de comunicación. Finalmente, se contempla la atención en el hogar de estos alumnos.

Basil C., y PUIG de la Bellacasa, R. (Eds.). (1988). Comunitación Aumentativa. Madrid: INSERSO.

Bustos, C. (1984). Reeducación del habla y el lenguaje en el paralítico cerebral. (2 $2^{\mathrm{a}}$ Ed.). Madrid: CEPE.

CASCÓN, P., y MARTÍN, C. (Eds.). (1989). La alternativa del juego. Fichas Técnicas. (8 Ed.). Cantabria: Colectivo educar para la paz. (Se puede obtener en : Colectivo educar para la paz. Aptdo. de Correos 219, Torrelavega, Cantabria).

FINNE, R. N. (1987). Atención en el bogar del niño con parálisis cerebral. Madrid: La Prensa Médica Mejicana.

HALE, (1985). Manual para el minusválido. Barcelona: Blume

MUSSELWHITE, C. R. (1990). Juegos adaptados para niños con necesidades especiales. Madrid: INSERSO. Colección Rehabilitación, 34.

Soro, E.; Roseli., C.; Alsina, G.; Garcla, H.; Sánchez de Muniaín, P.; Comellas, A.; Vilaseca, R. M., y EDO, S. (1988). Manual de toma de decisiones y de evaluación para el aprendizaje y uso de los sistemas aumentativos de comunicación. Serie: Evaluación. Documento I. Madrid: ATAM-FUNDESCO. (Se puede obtener en : Nadís, Canàries 1, 08017 Barcelona).

TOLEDO, M. (1984). La escuela ordinaria ante el niño con necesidades especiales. Madrid: Santillana-Aula XXI. VV. AA. (1990). Las necesidades educativas especiales del niño con deficiencia motora. Madrid: MEC-Centro Nacional de Recursos para la Educación Especial. Serie Formación.

VON TETZCHNER, S., y MARTINSEN, H. (1993). Introducción a la enseñanza de signos y al uso de ayudas técnicas para la comunicación. Madrid: Visor.

\section{RE'TRASO MENTAL}

El conjunto de referencias que se ofrecen pretenden abordar los problemas más relevantes que la educación de los alumnos con retraso mental plantea a los profesores. Tales problemas tienen que ver, fundamentalmente, con la nueva concepción de la educación especial y su reflejo en la actitud de los docentes y en la organización/función de los centros, particularmente los de educación especial, y con la necesidad de adaptar el currículo asegurando la funcionalidad de los aprendizajes a lo largo de toda la escolarización de dichos alumnos. Se introduce también un tema ciertamente novedoso en este campo como son las estrategias de aprendizaje:

ASHMAN, A. F., y CONWAY, R. (1990). Estrategias cognitivas en educación especial. Madrid: Aula XXI, Santillana.

BreCHIN, A., y SwaIn, J. (1990). Cambio de relaciones. Barcelona: Ediciones Milán. 
BRENNAN, W. K. (1988). El currículo para niños con necesidades esperiales. Madrid: M.E.C. y Siglo XXI.

BRoWN, L. (1989). Criterios de funcionalidad. Barcelona: Ediciones Milán.

DONLON, E. T., y Futron-BuRTONB, L. (1989). La enseñanza de los deficientes severos y profundos. Madrid: M.E.C. y Siglo XXI.

Gine, C.; Carasa, P.; FernÁndez, M.; Madurelu, J.; Ruiz, R., y Tirado, V. (1989). Educació especial. Noves perpectives. Barcelona: Editorial Laia. (Versión en castellano en prensa).

INGALLS, R. P. (1982). Retraso mental. La nueva perspectiva. México: Editorial El Manual Moderno.

LOVAAS, I. (1990). Enseñanza de niños con trastornos del desarrollo. Barcelona: Martínez Roca.

Ruiz, R. (1988). Técnicas de individualización didáctica. Adecuaciones curriculares individualizadas para alumnos con necesidades especiales. Madrid:Editorial Cincel.

VV. AA. (1991). El alumno con retraso mental en la escuela ordinaria. Madrid: M.E.C.-Centro Nacional de Recursos para la Educación Especial. Serie Formación.

\section{GRAVE RETRASO EN EL DESARROLLO}

La selección de las obras que a continuación se sugieren está presidida por la voluntad de ofrecer a los profesores recursos para la educación de los alumnos con grave retraso en el desarrollo, facilitando así la superación de las frecuentes barreras psicológicas; voluntad que responde al convencimiento de que es necesario sustraer a dichos alumnos de un modelo de atención basado únicamente en la asistencia. Las publicaciones se centran en dos ámbitos: sugerencias para la intervención educativa, sobre todo centrada en el area de la autonomía personal, y las posibilidades - y límites- de los sistemas alternativos de comunicación en la educación de estos alumnos.

KIERNAN, C.; JoRdAN, R., y SAUNDERS, C. (1978). Starting Off, London: Souvenir Press. Traducción Española: (1983) Cómo conseguir que el niño juegue y se comunique. Madrid: INSERSO.

MiLES, M. (1988): La deficiencia mental profunda: el último desafio. Siglo Cero, 116, Marzo-Abril, 1656.

MitTler, P. (1988): La ayuda a las personas con deficiencia profunda: Panorámica General. Siglo Cero, 116, Marzo-Abril, 12-15.

TAMARIT, J. (1988). Los trastornos de la comunicación en deficiencia mental y otras alteraciones evolutivas: intervención mediante sistemas de comunicación total. En Basil, C. y Puig, R. (Eds). Comunicación Aumentativa. Madrid: INSERSO.

TAMARIT, J. (1989). Uso y abuso de los sistemas alternativos de comunicación. Comunicación, Lenguaje y Educación, $n^{\circ} 1,81-94$.

VV. AA. (1991). Los problemas de comportamiento en Personas con Retraso Mental Grave. Zerbitzuan (Revista de Servicios Sociales del Consejo Vasco de Bienestar Sacial), $n^{\circ} 17$.

VV. AA. (1989). Intervención Educativa en Autismo Infantil. Madrid: M.E.C.-Centro Nacional de Recursos para la Educación Especial. Serie Formación. 